\title{
Canadian Society for Aesthetic (Cosmetic) Plastic Surgery/ Société canadienne de chirurgie plastique esthétique
}

\author{
Abstracts presented at the 38th Annual Meeting \\ September 16 and 17, 2011 \\ Program Chairpeople: \\ Dr Elizabeth Hall-Findlay, President \\ Dr Félix-André Têtu, Vice-President
}

1

\section{SUPRA-AREOLAR LIFT}

\section{G Waslen}

\section{Calgary, Alberta}

Supra-areolar lift is a surgical technique to elevate the nipple areolar complex. This is best suited for correction of nipple areola complex elevation asymmetries, and first- and possible second-degree breast ptosis. It can be combined with breast augmentation or performed following breast augmentation, or simply as a nipple areola lift.

The surgical procedure involves a skin incision at the superior areolar skin junction, with undermining of the superior skin to the predetermined new nipple areolar placement. A transverse incision is made superiorly to the areola, through the breast tissue to the pectoralis. A vertical incision is made in the superior breast tissue creating an inverted $\mathrm{T}$ incision in the breast tissue. Breast augmentation can be done through this incision. Closure of the breast is done with a Y-V closure, elevating the breast tissue. The excessive superior skin is excised and closure is accomplished with superior circum-areolar skin closure.

The surgical technique, indications, complications and pre and post examples will be shown and discussed in the presentation.

2

IMPROVING PERIORBITAL APPEARANCE IN THE NEGATIVE VECTOR PATIENT

\section{$M$ Yaremchuk}

Boston, Massachusetts, USA

Narrow palpebral fissures, short lower lids and full cheeks are hallmarks of youthful periorbita. The presence of these features is predicated on a convex upper midface skeleton. Faces whose midface skeletons are flat or concave (negative vector) do not manifest these youthful attributes, tend to age prematurely, and are prone to lower lid malposition after blepharoplasty.

Augmentation of the infraorbital rim with alloplastic implants can provide convexity to the deficient upper midface skeleton. Suspension of the cheek soft tissues (subperiosteal midface lift) on this now supportive framework narrows the palpebral fissure, shortens the lower lid and gives fullness to the cheek. The addition of lateral canthopexy to skeletal augmentation and subperiosteal midface lift can restore lower lid position when previous blepharoplasty has resulted in lower lid malposition in patients with deficient midface skeletons.

This concept has been employed in 87 patients ( 65 female, 22 male) in a recent 7 -year period. Of these 87 patients, 4 patients $(5 \%)$ required revision surgery to correct implant malposition or prominence. 3 patients $(3 \%)$ required implant removal to treat infection. Implants were later replaced in 2 of these 3 patients.

Augmentation of the infraorbital rim with alloplastic implants provides convexity to the upper midface skeleton. Together with lower lid and midface soft tissue suspension, it creates or restores youthful periorbital aesthetics.
3

\section{CREATING FACIAL BALANCE BY ENLARGING THE DEFICIENT LOWER JAW}

M Yaremchuk

Boston, Massachusetts, USA

Patients with small lower jaws may derive aesthetic benefit from alloplastic augmentation of both their anterior and posterior mandible. Augmentation of the contours of their mandible "balances" their facial dimensions while adding definition and angularity to the lower third of their face.

Most patients with small mandibles have a normal occlusion. Significant mandibular deficiency may result in Class II dental malocclusion. The majority of these patients can have their dental relationships normalized through orthodontic tooth movement. Those with severe mandibular deficiency require surgical advancement of the mandible to correct the malocclusion. The classic method of correcting Class II dental malocclusion in patients with significant mandibular deficiency, in addition to preoperative and postoperative orthodontic treatment, includes sagittal split ramus osteotomy and sliding advancement genioplasty, with possible LeFort I maxillary impaction. This combination of procedures can provide a Class I dental relationship while normalizing the skeletal contour. In patients with mandibular deficiency who have had their malocclusion corrected through orthodontics alone, mandibular osteotomy could disturb these dental relationships, requiring extensive perioperative orthodontic treatment. This type of treatment is both costly and time consuming. Alloplastic augmentation of the mandible can provide a visual effect similar to, and, in my opinion, superior to that of sagittal osteotomy with advancement.

This lecture presents my operative strategies for improving the skeletal contours of the deficient mandible with normal occlusion. The anatomy associated with a deficient mandible, that can be camouflaged with implants, includes the obtuse mandible angle with steep mentocervical angle, the decreased vertical and transverse ramus dimensions as well as the poorly projecting chin. When the chin is deficient in both the vertical and sagittal dimensions, it is lengthened vertically by horizontal osteotomy and augmented sagitally with an implant. When the poorly projecting chin is long in the vertical direction, it is shortened, and then augmented sagitally with an implant.

In addition to normalizing dimensions of the deficient mandible, modest augmentation of lower jaws with normal dimensions can help balance upper and lower facial relations as well as increase angularity.

\section{4}

PRIMARY RHINOPLASTY USING DICED CARTILAGE AND FASCIA GRAFTS

J Calvert

Beverly Hills, California, USA

Primary rhinoplasty can require grafting of the radix and dorsum to create the look the patient desires. Diced cartilage and deep temporalis fascia has emerged as a useful tool in adding structure and contour to these key areas. In order to utilize this technique effectively, it is necessary to perform a full examination of the patient, make a proper diagnosis of the problems with the nose, perform a detailed analysis of the structural and aesthetic situation, and then choose the technique for grafting appropriately. Implementation of the technique is not trivial and complications do occur 
that vary in type and magnitude. This discussion will enumerate these aspects of the technique such that the attending surgeon may gain an understanding and utilize the method of grafting in primary rhinoplasty.

\section{5}

\section{REVISION RHINOPLASTY USING RIB GRAFTS \\ J Calvert}

Beverly Hills, California, USA

Secondary rhinoplasty is very different than primary rhinoplasty in that the nose and the patient have both already been through previous surgery and are scarred in some manner from the previous experience. Reoperation is technically more difficult and the need for grafting the nose with cartilage from sources other than septum makes the operation that much more complex. Rib cartilage provides an abundant source of material that is generally more reliably strong, easy to shape and carve, and creates better long-term results when used properly. However, many surgeons feel that it is difficult to obtain, has problems with warping and shape problems, and that patients do not want to have their chest violated for their nose. This discussion will explore these concepts and provide the operating surgeon with ideas on how to incorporate rib cartilage grafts into their secondary rhinoplasty practice.

\section{6}

MAMMAPLASTIES: WHICH OPERATION WHEN AND

\section{HOW?}

\section{N Corduff}

\section{Geelong, Australia}

The key to consistently good results in this area of aesthetic surgery is analysis of the problem and then careful consideration of the appropriate techniques for surgical correction. One technique will give good results in many cases, but will also give less than optimum outcomes in other cases. I believe that this area needs more than one approach; a surgeon who applies only one technique to reduction mammaplasty is at a disadvantage. One needs to be familiar with all mammaplasty techniques and be versatile with their use to give consistently good results.

Understanding the vascular anatomy of the breast is essential in determining surgical technique. From the main arterial supply, a dominant blood supply develops around the breast. As it develops, it pushes into the subcutaneous tissue dragging a blood supply outside the breast capsule, which becomes more superficial as it reaches a subareolar plexus and then feeds retrogradely into the breast gland. The venous circulation is in the reverse direction.

In assessing a hypertrophic breast, if the patient is young, has a good base shape, minimal ptosis, good skin and good nipple position on the breast mound, then a subglandular approach is appropriate. This avoids visible scars, which can be devastating to the young teenager. Parenchyma can be resected from behind and within the breast without skin resection. The excess skin will take up around the reduced breast mound in these young patients.

The majority of breast reduction patients will be older when the supporting ligamentous structure of the breast becomes stretched and the heavy breast descends down the chest wall. With pregnancy and breast feeding, as the gland rapidly enlarges, the base diameter of the breast enlarges and expands medially, inferiorly and laterally. To create an aesthetic breast shape, the tissue needs to be resected to reduce the breast base diameter back to within the aesthetic boundaries of the anterior axillary line and lateral sternal edge. When the breast is 'unloaded', the inframammary crease will lift and form a pleasing arc to the lower pole.

There usually needs to be volume restoration to the upper pole, giving central projection to the new shape.

Assessment of where the tissue needs to be resected is important. The parenchymal tissue left behind needs to be considered as multiple vascularised flaps. Understanding the vascular anatomy allows appropriate technique selection. The flap carrying the nipple areola complex needs to avoid kinking of its vascular supply and not be too long. Thus, the position of the nipple areolar complex on the breast mound pre-operatively will often determine whether it is carried on a superior, supero-medial or inferior pedicle. In extremely large breasts, it is not unreasonable to consider transferring the nipple areolar complex as a free nipple graft.

Finally, skin resection and closure needs to be considered separately to the technique used to translocate the nipple areolar complex. By tailoring the skin resection to the new mound at the end of the procedure, the wounds can be closed with reduced tension and the scars shortened and placed in the optimum position.

\section{7}

\section{LARGE BREAST AUGMENTATION - LESIONS LEARNED \\ K Rai}

New Westminster, British Columbia

INTRODUCTION: Augmentations ranging from 500 ccs to $1400 \mathrm{ccs}$ are discussed and presented. The patients who request this correction of large breast augmentation are usually well informed, extremely fussy and have high expectations.

Augmentations are done usually in the subpectoral plane, but some patients prefer a pre-pectoral position. Augmentation is done up to $800 \mathrm{ccs}$; a style 20 gel is preferred since it is a high profile implant, giving them the desired fullness in the upper pole. For patients wanting over $800 \mathrm{ccs}$, the preference is to use a moderate profile saline implant and increase the volume beyond its capacity to fill up to 1000,1200 to 1400 ccs.

Breast implants over $1000 \mathrm{ccs}$ are not recommended in the prepectoral plane since subsequent ptosis of the breast is an issue. The subpectoral plane is preferred in the larger implants. Follow-ups do show satisfactory results.

The technique with these large implants is either transaxillary or preferably the infra-areolar approach, with the implants placed in the subpectoral plane.

RESULTS: We have not seen any rupture of the implant. The rippling effect is minimal when the implants are overinflated. The satisfaction results have been very high.

CONCLUSION: A group of women prefer overfilled large implants to give them prepectoral fullness and a large breast look, which gives them a voluptuous, sexy look. They have very high expectations. Long-term follow-ups have shown no rippling within these large implants that have been over-filled (saline implants). Saline implants are preferred over $800 \mathrm{ccs}$ to $1400 \mathrm{ccs}$. Initially the implants are usually tense and firm, but usually soften up within 3 to 4 months. We have not seen any stretch marks in these patients.

\section{8}

\section{COSMETIC SURGERY IN CANADIAN PLASTIC SURGERY RESIDENCIES: ARE WE TRAINING COMPETENT SURGEONS Q Chivers, A Arkoubi, K Murray, A Islur \\ Winnipeg, Manitoba}

PURPOSE: A large majority of plastic surgeons receive minimal exposure to cosmetic surgery during their residency training. This is the conclusion of recent research published by the American Society of Plastic Surgeons. A similar situation is thought to exist in Canada. As cosmetic surgery becomes marginalized to private surgery centres and fewer academic staff perform aesthetic surgery, residents are less likely to gain exposure to the field given the increasingly busy traumatic and reconstructive service requirements at most of the major teaching hospitals. To better assess cosmetic surgery exposure in plastic surgery residency programs, graduating residents were asked to record their impressions of cosmetic surgery training in each of their respective programs.

METHODS: All graduating residents enrolled in plastic surgery residency programs in Canada were surveyed with a short questionnaire. The survey addressed two broad areas: (1) specifics regarding resident cosmetic surgery training and (2) the confidence and satisfaction associated with this experience.

RESULTS: A majority of Canadian plastic surgery residency programs currently have a designated cosmetic surgery rotation. Most training however, comes from staff without a significant cosmetic surgery practice, with residents typically performing $<20 \%$ of the cases. $90 \%$ of respondents feel it has become increasingly difficult to see cosmetic surgery cases due to a higher number of them being performed at private surgical centres, with $62 \%$ believing training could be improved through increased access to cosmetics cases performed at major teaching hospitals. Outside electives performed in cosmetic surgery were felt to be the most beneficial source of learning overall. Many residents describe lower than average confidence in performing several cosmetic surgery procedures upon completion of their residency training. Most feel a need to pursue additional training prior to being able to incorporate cosmetic surgery into their practice, which many are in fact not pursuing. 
CONCLUSIONS: RCPSC certification in plastic surgery does not necessarily equal proven competency in cosmetic surgery despite this subspecialty accounting for $1 / 5$ th of the examination process. To maintain the specialty's reputation as a leader in the field and ensure its members proficiency in this continually evolving and competitive market, Canadian plastic surgery programs need to ensure their residents receive adequate exposure to cosmetic surgery procedures and technologies.

*Morrison, CM; Rotemberg, SC; Moreira-Gonzalez, A; Zins, J. A Survey of Cosmetic Surgery Training in Plastic Surgery Programs in the United States. Plastic and Reconstructive Surgery: 2008;122(5);1570-1578.

9

CAPSULAR IMPLANTS AND IMMUNOPHENOTYPES OF

\section{INFLAMMATORY CELLS}

EM Meza Britez, Carmelo Caballero

Asuncion, Paraguay

It is mandatory to study the capsule when we have to replace the implant, with or without capsular contracture, as surgical biopsy material.

Immunohistochemistry and certain studies through molecular pathology can help the plastic surgeon understand new concepts about other immune system diseases and abnormal proliferation of cells such as the growth of neoplastic cells.

\section{0}

\section{DOES KNOWLEDGE OF THE INITIAL TECHNIQUE AFFECT} OUTCOMES AFTER REPEAT BREAST REDUCTION

\section{J Ahmad, SM Mclsaac, F Lista}

\section{Mississauga, Ontario}

Repeat breast reduction has been approached with great apprehension in the literature. Outcomes following repeat breast reduction using our approach for vertical scar reduction mammaplasty were retrospectively reviewed. All patients who underwent repeat breast reduction for recurrent symptomatic mammary hypertrophy, inadequate volume reduction during the primary surgery, and significant postoperative breast volume asymmetry were included. The safety of performing this procedure in patients for whom operative records were available for the previous breast reduction was compared with those for whom these records could not be obtained. Twenty-five patients had repeat breast reduction. The initial technique was known in 13 patients and unknown in 12 patients. The average total reduction per breast (including liposuction) was $658 \mathrm{~g}$ (range 30 to $1150 \mathrm{~g}$ ). Liposuction was used more often in cases in which the initial technique was unknown $(\mathrm{P}=0.000)$. No patients experienced necrosis of the nipple-areola complex, and there was no significant difference in the complication rates between patients for whom the previous pedicle was known versus those in whom it was unknown $(\mathrm{P}=0.220)$. In summary, repeat breast reduction with vertical scar reduction mammaplasty is safe, even when the initial technique is unknown. A vertically oriented, inferior wedge excision of tissue can be safely excised, irrespective of the initial pedicle. For patients with ptosis in whom the nipple-areola complex needs to be transposed superiorly, a carefully planned and de-epithelialized superior pedicle should be used. In addition, liposuction is an important adjunct to achieve volume reduction while limiting the amount of dissection during repeat breast reduction.

\section{1}

BIOPHOTONICS IN FACIAL REJUVENATION. A PROSPECTIVE RANDOMIZED INVESTIGATIVE TRIAL

A Nikolis, S Bernstein, S Rastogi, JS Sampalis

Montreal, Quebec

INTRODUCTION: Established techniques in the non-surgical management of facial aesthetics frequently involve the use of injectables, lasers or peels and various radiofrequency devices. To date, the benefit from wavelength-specific LED light with a chromophore-rich emollient has not been previously investigated in facial aesthetics. The purpose of the study is to evaluate whether blue light in combination with a chromophorerich emollient impacts facial aesthetics.

MATERIALS AND METHODS: Following scientific/ethics committee approval, 32 female participants were randomized into a split face trial evaluating the use of emollient and light (EL) against light alone (L), emollient alone (E) or a baseline retinol cream (R). Participants were treated at weekly intervals over a one month period, and followed for six months with clinical evaluations, digital photography and tissue biopsies. Photographs were evaluated by a three-surgeon blinded committee using established facial scoring systems. Participant perceptions and outcomes were also analyzed.

RESULTS: Thirty of the 32 participants completed the trial. There were no serious adverse events reported. Participants in the EL group demonstrated statistically significant improvements in the crow's feet and brow regions, while participants in the $\mathrm{L}$ group demonstrated improvement in the brow region. Participant satisfaction was statistically significant in the EL and L groups. Gomori-Trichrome staining demonstrated a six-fold increase in the density of collagen fascicles in the EL group.

CONCLUSION: The combination of blue light with a chromophore-rich emollient in a non-interacting carbomer improves facial aesthetics in multiple facial subunits. Further evaluation of this technology is warranted.

\section{AUTHOR INDEX}

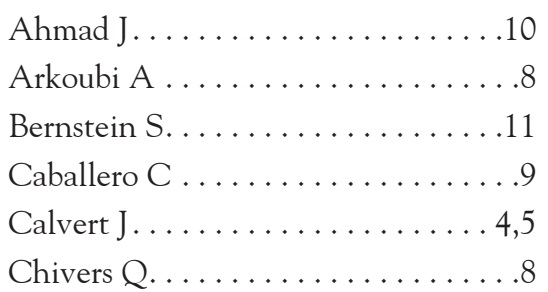

Corduff N..............6

Islur A ................ . 8

Lista F. . . . . . . . . . . . . . . . 10

McIsaac SM . . . . . . . . . . . . . . 10

Meza Britez EM . . . . . . . . . . . . . .9

Murray K . . . . . . . . . . .8
Nikolis A ................11

Rai K ................

Rastogi S ..................

Sampalis..................11

Waslen $\mathrm{G} \ldots \ldots \ldots \ldots \ldots \ldots \ldots$

Yaremchuk M............. 2,3 